\title{
Coloração das gemas de ovos de poedeiras alimentadas com ração à base de sorgo com adição de pigmentantes naturais e sintéticos
}

\author{
Coloring of egg yolks of laying hens fed with sorghum-based feed with addition of natural \\ and synthetic pigments
}

Diogo Alvarenga Miranda 1 (ORCID 0000-0001-9576-8810), Jean Kaique Valentim 2 (ORCID 0000-0001-8547-4149), Helder Freitas de Oliveira $3^{\star}$ (ORCID 0000-0003-4109-1087), Claudiane Aparecida Rocha Chaves 4 (ORCID 0000-0001-6183-1578), Adriano Geraldo 4 (ORCID 0000-0001-5342-6863), Luiz Carlos Machado 4 (ORCID 0000-0003-4126-9535), Janaína Palermo Mendes 5 (ORCID 0000-0002-7860-0933), Alexander Alexandre de Almeida ${ }^{1}$ (ORCID 0000-0001-7313-4008)

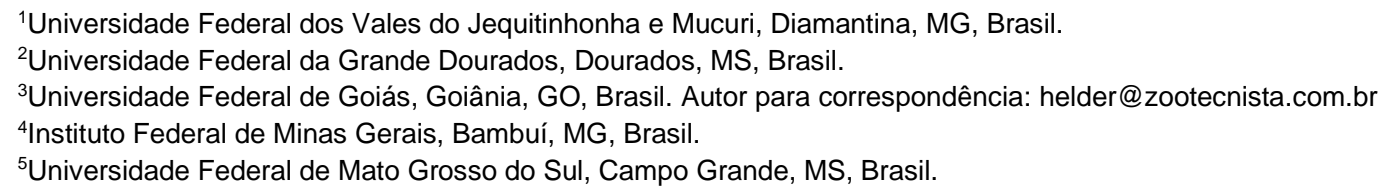

Submissão: 31/08/2021 | Aceite: 13/10/2021

\begin{abstract}
RESUMO
Objetivou-se avaliar a inclusão de pigmentante sintético (Cantaxantina 10\%) aliado a níveis de suplementação de pigmentantes naturais e sintéticos na dieta de poedeiras comerciais em rações a base de sorgo. Foram utilizadas 150 poedeiras comerciais com 67 semanas de idade da linhagem Hisex Brown, distribuídas em delineamento inteiramente casualizado com cinco tratamentos, seis repetições de cinco aves/repetição. Os tratamentos avaliados foram: $25 \mathrm{~g} / \mathrm{t}$ de carophyll yellow $10 \%+20 \mathrm{~g} / \mathrm{t}$ de carophyll vermelho (T1); $300 \mathrm{~g} / \mathrm{t}$ de Marigold 2\% + $10 \mathrm{~g} / \mathrm{t}$ de Cantaxantina 10\% (T2); $300 \mathrm{~g} / \mathrm{t} \mathrm{de} \mathrm{Marigold} \mathrm{2 \%} \mathrm{+} 20 \mathrm{~g} / \mathrm{t}$ de Cantaxantina 10\% (T3); $300 \mathrm{~g} / \mathrm{t}$ de Marigold 2\% + $30 \mathrm{~g} / \mathrm{t}$ de Cantaxantina 10\% (T4); $300 \mathrm{~g} / \mathrm{t}$ de Marigold $2 \%+20 \mathrm{~g} / \mathrm{t}$ carophyll vermelho (T5). Foi aplicado o teste de Tukey ao nível de 5\% de probabilidade para as variáveis de desempenho e qualidade de ovos. Houve diferença entre os tratamentos apenas para coloração da gema, onde o aumento da coloração teve reflexo de acordo com o aumento dos níveis de pigmentante nas dietas, sendo que as demais variáveis não foram afetadas. O nível de suplementação de 300 g.t $\mathrm{t}^{-1}$ de pigmento natural amarelo $+30 \mathrm{~g}^{\mathrm{t}^{-1}}$ de pigmento vermelho industrial em dietas a base de sorgo para poedeiras proporcionou melhor índice de coloração da gema.
\end{abstract}

PALAVRAS-CHAVE: aceitação do consumidor, carotenoides, cor de gema, extratos vegetais.

\begin{abstract}
The objective of this study was to evaluate the inclusion of synthetic pigment (Cantaxanthin 10\%) combined with levels of natural and synthetic pigment supplementation in the diet of commercial laying hens in diets based on sorb. We used 150 commercial laying hens with 67 weeks of age of the Hisex Brown strain, distributed in a completely randomized design with five treatments, six replicates of five birds/repetition. The treatments evaluated were: $25 \mathrm{~g} / \mathrm{t}$ carophyll yellow $10 \%+20 \mathrm{~g} / \mathrm{t}$ red carophyll (T1); $300 \mathrm{~g} / \mathrm{t}$ of Marigold 2\% $+10 \mathrm{~g} / \mathrm{t}$ of Cantaxanthin 10\% (T2); $300 \mathrm{~g} / \mathrm{t}$ of Marigold $2 \%+20 \mathrm{~g} / \mathrm{t}$ of Cantaxanthin $10 \%$ (T3); $300 \mathrm{~g} / \mathrm{t}$ of Marigold $2 \%+30 \mathrm{~g} / \mathrm{t}$ of Cantaxanthin $10 \%$ (T4); $300 \mathrm{~g} / \mathrm{t}$ of Marigold $2 \%+20 \mathrm{~g} / \mathrm{t} \mathrm{red}$ carophyll (T5). The Tukey test was applied at the level of $5 \%$ probability for the various performance and quality of eggs. There was a difference between treatments only for yolk staining, where the increase in color was reflected according to the increase in pigmenting levels in the diets, and the other variables were not affected. The supplementation level of 300 g.t- 1 of natural yellow pigment $+30 \mathrm{~g} . \mathrm{t}-1$ of industrial red pigment in diets based on sorb for laying hens provided better yolk color index.
\end{abstract}

KEYWORDS: consumer acceptance, carotenoids, yolk color, vegetable extract.

\section{INTRODUÇÃO}

O Brasil destaca-se no cenário internacional em relação à produção de proteína animal, sendo que a produção de ovos ocupa a sétima colocação dentre os países produtores do mundo (ABPA 2020). Em 2019, 
cerca de $66,59 \%$ da produção de ovos foi destinada ao mercado interno e 0,41\% destinada à exportação (cerca de 7.698 toneladas). Para o mercado interno, o consumo per capita foi de 230 ovos no mesmo ano (ABPA 2020).

Para que o setor avícola continue se desenvolvendo cada vez mais, pesquisas são realizadas a fim de proporcionar maior qualidade do produto final. A coloração da gema dos ovos é avaliada por meio da deposição de xantofilas, pigmentos carotenoides derivados da alimentação das aves. O milho é a principal fonte de pigmento amarelo em rações comerciais, portanto, ao substituí-lo por alimentos alternativos, como por exemplo, o sorgo e o milheto, a pigmentação da gema é alterada (BITTENCOURT et al. 2019).

A qualidade dos ovos é influenciada pela nutrição e seus aspectos visíveis como cor de gema e aparência da casca são parâmetros observados pelos consumidores, remetendo a cuidados relacionados com a alimentação destas aves quando há substituição total do milho por alimentos pobres em carotenos nas rações de galinhas poedeiras. Quando há redução da intensidade na cor da gema é interessante a inclusão de pigmentantes na ração para solucionar esse problema (MOURA et al. 2011, LANA et al. 2017).

A maioria dos componentes do ovo é metabolizada no fígado e o acúmulo de nutrientes na circulação sanguínea faz com que sejam transportados até o ovário, onde ocorre a deposição dos compostos lipossolúveis, lipídios, fosfolipídios, colesterol e os carotenoides que dão a cor amarelo-laranja da gema (LOPES et al. 2011).

A cor da gema é uma característica de relevância econômica por ser associada à sua qualidade nutricional, mesmo não tendo relação entre tais aspectos. Tratando-se de aves poedeiras, deve-se considerar que mudanças químicas e nutricionais em ovos podem ocorrer por meio da adição de determinados compostos à dieta. A presença de carotenóides nos alimentos consumidos influencia a intensidade de coloração da gema (SEIBEL et al. 2010, OLIVEIRA et al. 2021), utilizando na ração de codornas japonesas, extrato produzido a partir do resíduo do processamento de goiaba, observaram que a inclusão do extrato propiciou um aumento na coloração da gema dos ovos.

A preferência pelo grau de pigmentação da gema varia entre os consumidores de diferentes países, ou mesmo entre regiões de um mesmo país. Nos Estados Unidos e no Brasil, o consumidor prefere colorações entre 7 e 10 na escala colorimétrica DSM (DYCF). Por outro lado, na Europa e Ásia, os consumidores têm preferência por gemas mais pigmentadas, entre 10 e 14 no DYCF (GALOBART et al. 2004). Devido às exigências de mercado, os pigmentantes naturais têm sido cada vez mais utilizados para potencializar a cor das gemas dos ovos. A utilização de alimentos alternativos é uma realidade na produção animal, devido a alta nos preços de insumos comumente utilizados como o milho e soja. Assim a utilização de sorgo em dietas para galinhas poedeiras apresenta diversas vantagens, pois é comercializado a um preço menor do que do milho, por apresentar diferenças nutricionais (FASSANI et al. 2019). Porém apresenta a desvantagem de proporcionar cor de gema em menor intensidade, por isso justifica-se utilizar pigmentantes associados as dietas de sorgo, sejam eles sintéticos ou naturais.

Com isso, o objetivo do trabalho é avaliar a inclusão de um pigmentante industrial a base de Cantaxantina a 10\% aliado a níveis de suplementação de pigmentantes naturais e sintéticos na dieta de poedeiras comerciais em rações formuladas a base de sorgo e seus efeitos sobre o desempenho produtivo, qualidade interna e externa dos ovos e a coloração da gema.

\section{MATERIAL E MÉTODOS}

O experimento foi conduzido no laboratório experimental de avicultura de postura do Instituto Federal de Minas Gerais (IFMG), Bambuí, Minas Gerais, Brasil. O projeto de pesquisa foi aprovado pelo Comitê de Ética no Uso de Animais (CEUA) do IFMG (protocolo no 05/2017).

Foram utilizadas 150 aves poedeiras com 67 semanas de idade da linhagem Hisex Brown, instaladas em galpão experimental, dotado de gaiolas com as dimensões individuais de $0,45 \mathrm{~m}$ largura $\times 0,50 \mathrm{~m}$ profundidade $\times 0,40 \mathrm{~m}$ altura com capacidade de alojar cinco aves em cada utilizando a densidade de 450 $\mathrm{cm}^{2} /$ ave. Cada gaiola contem bebedouro nipple e comedouro tipo calha. O pigmento carophyll yellow e carophyll vermelho são pigmentos sintéticos, já o Marigold $2 \%$ é considerado pigmento natural amarelo e Cantaxantina $10 \%$ um pigmento industrial vermelho. Foi adotado o programa de luz de 16 horas/dia e diariamente foi mensurado através de um termo higrômetro, as temperaturas e umidade relativa do ar a cada 15 minutos. As médias de temperatura obtidas no período experimental foi de $21,5^{\circ} \mathrm{C}$ e máxima de $29,7^{\circ} \mathrm{C}$ e umidade relativa do ar mínima de $49,6 \%$ e máxima de $76,6 \%$.

Foi adotado o delineamento inteiramente casualizado, com 5 tratamentos e 6 repetições com 5 aves por parcela, totalizando 30 parcelas experimentais, sendo realizado em 3 períodos de 28 dias, totalizando 84 dias experimentais. As aves foram alimentadas com rações experimentais à base de sorgo, farelo de 
soja, fosfato bicálcico, com suplementação de uma mistura de carboidrases (150 g/t com valorização de 75 kcal), emulsificante a base de lecitina hidrolisada de soja (250 g/t com valorização de $50 \mathrm{kcal})$, fitase (500 $\mathrm{U} / \mathrm{kg}$ e redução em 0,13 e 0,13 pontos porcentuais para fósforo disponível e cálcio, respectivamente), seguindo um padrão de exigência nutricional da linhagem. Todas as dietas foram isoproteicas, isoaminoacídicas, isocálcicas e isofosfórica (Tabela 1).

Os tratamentos avaliados foram: $25 \mathrm{~g} / \mathrm{t}$ de carophyll yellow $10 \%+20 \mathrm{~g} / \mathrm{t}$ de carophyll vermelho (T1); $300 \mathrm{~g} / \mathrm{t}$ de Marigold 2\% + $10 \mathrm{~g} / \mathrm{t}$ de Cantaxantina 10\% (T2); $300 \mathrm{~g} / \mathrm{t}$ de Marigold 2\% + $20 \mathrm{~g} / \mathrm{t}$ de Cantaxantina $10 \%$ (T3); $300 \mathrm{~g} / \mathrm{t}$ de Marigold 2\% + $30 \mathrm{~g} / \mathrm{t}$ de Cantaxantina 10\% (T4); $300 \mathrm{~g} / \mathrm{t}$ de Marigold 2\% +20 g/t carophyll vermelho (T5).

Tabela 1. Composição das dietas experimentais e teores nutricionais aplicados.

Table 1. Composition of experimental diets and applied nutritional contents.

\begin{tabular}{|c|c|c|c|c|c|}
\hline \multirow[b]{2}{*}{ Ingredientes } & \multicolumn{5}{|c|}{ Tratamentos } \\
\hline & T1 & T2 & T3 & T4 & T5 \\
\hline Sorgo & 68,07 & 68,07 & 68,07 & 68,07 & 68,07 \\
\hline Farelo de Soja 45.0 & 20,81 & 20,81 & 20,81 & 20,81 & 20,81 \\
\hline Fosfato Bicálcico & 0,640 & 0,64 & 0,64 & 0,64 & 0,64 \\
\hline Calcário 38.0 & 9,58 & 9,58 & 9,58 & 9,58 & 9,58 \\
\hline Cloreto de sódio & 0,39 & 0,39 & 0,39 & 0,39 & 0,39 \\
\hline L-Lisina 78.0 & 0,08 & 0,08 & 0,08 & 0,08 & 0,08 \\
\hline DL-Metionina 99.0 & 0,15 & 0,15 & 0,15 & 0,15 & 0,15 \\
\hline L-Treonina 98.0 & 0,02 & 0,02 & 0,02 & 0,02 & 0,02 \\
\hline HCL Betaína 93.0 & 0,03 & 0,03 & 0,03 & 0,03 & 0,03 \\
\hline Fitase $10.000 \mathrm{FTU}$ & 0,01 & 0,01 & 0,01 & 0,01 & 0,01 \\
\hline Premix vitamínico ${ }^{1}$ & 0,10 & 0,10 & 0,10 & 0,10 & 0,10 \\
\hline Premix mineral ${ }^{2}$ & 0,10 & 0,10 & 0,10 & 0,10 & 0,10 \\
\hline Marigold 2\% & 0,00 & 0,30 & 0,30 & 0,30 & 0,30 \\
\hline Carophyll vermelho 10\% & 0,025 & 0,00 & 0,00 & 0,00 & 0,025 \\
\hline Carophyll yellow $10 \%$ & 0,025 & 0,00 & 0,00 & 0,00 & 0,00 \\
\hline Cantaxantina $10 \%$ & 0,00 & 0,10 & 0,02 & 0,03 & 0,00 \\
\hline Enzima $^{4}$ & 0,0200 & 0,0200 & 0,02 & 0,02 & 0,02 \\
\hline \multicolumn{6}{|c|}{ Teores Nutricionais calculados } \\
\hline Proteína Bruta (\%) & 16,0 & 16,0 & 16,0 & 16,0 & 16,0 \\
\hline Extrato etéreo (\%) & 2,30 & 2,30 & 2,30 & 2,30 & 2,30 \\
\hline Fibra bruta (\%) & 2,80 & 2,80 & 2,80 & 2,80 & 2,80 \\
\hline Cálcio (\%) & 4,07 & 4,07 & 4,07 & 4,07 & 4,07 \\
\hline Fósforo total (\%) & 0,26 & 0,26 & 0,26 & 0,26 & 0,26 \\
\hline Fósforo disponível (\%) & 0,40 & 0,40 & 0,40 & 0,40 & 0,40 \\
\hline Sódio (\%) & 0,17 & 0,17 & 0,17 & 0,17 & 0,17 \\
\hline Cloro (\%) & 0,27 & 0,27 & 0,27 & 0,27 & 0,27 \\
\hline EMA disponível (kcal/kg) & 2780 & 2780 & 2780 & 2780 & 2780 \\
\hline Lisina total (\%) & 0,64 & 0,64 & 0,64 & 0,64 & 0,64 \\
\hline Lisina digestível (\%) & 0,71 & 0,71 & 0,71 & 0,71 & 0,71 \\
\hline Metionina total (\%) & 0,29 & 0,29 & 0,29 & 0,29 & 0,29 \\
\hline Metionina dig. (\%) & 0,36 & 0,36 & 0,36 & 0,36 & 0,36 \\
\hline Met+Cis total (\%) & 0,42 & 0,42 & 0,42 & 0,42 & 0,42 \\
\hline Met+Cis dig. (\%) & 0,55 & 0,55 & 0,55 & 0,55 & 0,55 \\
\hline Triptofano total (\%) & 0,14 & 0,14 & 0,14 & 0,14 & 0,14 \\
\hline Triptofano dig. (\%) & 0,17 & 0,17 & 0,17 & 0,17 & 0,17 \\
\hline Treonina total (\%) & 0,39 & 0,39 & 0,39 & 0,39 & 0,39 \\
\hline Treonina dig. (\%) & 0,50 & 0,50 & 0,50 & 0,50 & 0,50 \\
\hline
\end{tabular}

${ }^{1}$ Nível de garantia do premix vitamínico por kg de produto: Cálcio (Mín) $170 \mathrm{~g}$, Cálcio (Máx) $210 \mathrm{~g}$, Fósforo (Mín) $45 \mathrm{~g}$, Metionina (Mín) 10 g, Colina (Mín) 5000 mg, Sódio (Mín) 30 g, Vitamina A (Mín) 140000 U.l, Vitamina D3 (Mín) 35000 U.I, Vitamina E (Mín) 140 U.I, Vitamina K3 (Mín) 30 mg, Tiamina B1 (Mín) 10 mg, Riboflavina B2 (Mín) 75 mg, Piroxidina B6 (Mín) $20 \mathrm{mg}$, Vitamina B12 (Mín) $120 \mathrm{mcg}$, Ácido Fólico (Mín) $6 \mathrm{mg}$, Niacina (Mín) $300 \mathrm{mg}$, Pantotenato de Cálcio (Mín) ${ }^{2}$ Nível de garantia do premix mineral por kg do produto: $120 \mathrm{mg}$, Cobre (Mín) $160 \mathrm{mg}$, Ferro (Mín) $630 \mathrm{mg}$, lodo (Mín) 20 mg, Manganês (Mín) 1600 mg, Selênio (Mín) 6 mg, Zinco (Mín) 1300 mg, Fitase (Mín) 10000 FTU, Bacitracina de Zinco 500 mg. ${ }^{3}$ Nível de garantia do Pigmentante Natural: Carotenoides (Mín.) 20,0g/kg. Nível de garantia Pigmentante Sintético: 20-40 ppm de CAROPHYLL® yellow. ${ }^{4}$ Composição Enzima U/g: Xilanase $>10000$. Protease $\geq$ 4000. Amilase $\geq 800$. Beta Mananase $\geq 1000$. Betaglucanase $\geq 700$. Ala Galactose $\geq 100$. 
Ao final de cada período de 28 dias, avaliaram-se o consumo de ração (g/ave/dia), produção de ovos (\%), massa de ovos (kg), conversão alimentar por massa de ovos $(\mathrm{kg} / \mathrm{kg})$, e por dúzias de ovos $(\mathrm{kg} / \mathrm{dz})$, para avaliação do desempenho produtivo das aves. Para análise da qualidade dos ovos, nos últimos três dias de cada período foram selecionados aleatoriamente dois ovos por repetição, onde se avaliou: peso médio do ovo, espessura da casca $(\mathrm{mm})$, cor da gema (escore de 1 a 15), gravidade específica, unidade Haugh, e porcentagens (\%) de albúmen, casca e gema.

A produção dos ovos em porcentagem foi calculada dividindo-se a quantidade total de ovos por parcela no período pelo número de aves. O consumo de ração ( $\mathrm{g} / \mathrm{ave} / \mathrm{dia}$ ) foi calculado pela diferença de peso da ração fornecida e peso das sobras, no $1^{\circ}$ e $28^{\circ}$ dia do ciclo, respectivamente, de cada unidade experimental. O cálculo da massa de ovo foi realizado pela multiplicação da produção de ovos (unidade) pelo peso médio dos ovos $(\mathrm{g})$ por parcela. A conversão alimentar por massa de ovo foi calculada pela relação entre consumo de ração e massa de ovo produzida. A conversão por dúzia de ovos foi calculada como a relação entre o consumo de ração e a produção; e o resultado multiplicado por 12.

Para análise da qualidade, dois ovos foram identificados e pesados em balança eletrônica de precisão $( \pm 0,001 \mathrm{~g})$. Após identificados e pesados, foi determinada a gravidade específica (GE) seguindo metodologia descrita por FREITAS (2004), os ovos foram pesados no ar e na água destilada, com controle da temperatura da água. A GE foi calculada por meio da divisão do peso dos ovos no ar pelo peso dos ovos na água. Após determinação da GE os ovos foram quebrados e seu conteúdo (albúmen + gema), colocado em superfície de vidro plana e nivelada, obtendo-se com auxílio de um micrômetro as medidas de altura de albúmen (mm). O ponto usado como referência para determinação da altura do albúmen foi a região entre a borda externa da gema perpendicular à chalaça, esta medida foi utilizada para fins de cálculo da unidade Haugh, utilizando a equação unidade Haugh utilizou-se a equação: $U H=100 \times \log \left(H-1,7 \mathrm{P}^{0,37}+7,57\right)$, onde: $\mathrm{UH}$ = unidade Haugh; $\mathrm{H}$ = altura do albúmen; $\mathrm{P}$ = peso do ovo (ALLEONI \& ANTUNES 2001).

A determinação da cor da gema foi realizada com a utilização do leque colorimétrico de Roche ${ }^{\circledR}$. A espessura da casca foi obtida com a utilização de paquímetro digital em dois pontos na área centrotransversalda casca do ovo, obtendo-se a média, expressa em milímetros $(\mathrm{mm})$ para análise estatística.

Os dados foram verificados quanto a normalidade dos resíduos utilizando-se o teste de Shapiro-Wilk e homogeneidade das variâncias com uso do teste de Levene. Posteriormente, foram submetidos a análise de variância pelo programa SISVAR. Quando observado efeito significativo foram realizadas comparações de médias com uso do teste de Tukey. Adotou-se $\alpha=0,05$.

\section{RESULTADOS E DISCUSSÃO}

Não houve diferenças significativas $(p>0,05)$ sobre as avaliações de desempenho (consumo, produção e conversão alimentar) entre os tratamentos com pigmentantes utilizados neste estudo (Tabela 2).

Tabela 2. Desempenho de galinhas poedeiras comerciais recebendo dietas com diferentes níveis de suplementação de pigmentantes sintéticos e naturais em dietas a base de sorgo.

Table 2. Performance of commercial laying hens fed diets with different levels of synthetic and natural pigment supplementation in sorghum-based diets.

\begin{tabular}{llllllll}
\hline \multirow{2}{*}{ Variáveis } & \multicolumn{7}{c}{ Tratamentos } \\
\cline { 2 - 8 } & T1 & T2 & T3 & T4 & T5 & CV & P-valor \\
\hline Consumo ração/ave/dia (g) & 106 & 108 & 109 & 104 & 110 & 3,25 & 0,23 \\
Produção ovos/ave/dia (\%) & 84,6 & 90,9 & 86,3 & 80,0 & 89,7 & 3,19 & 0,14 \\
CA (kg de ração/dúzia de Ovos) & 1,55 & 1,44 & 1,55 & 1,58 & 1,50 & 4,84 & 0,07 \\
CA (kg de ração/kg de ovos) & 1,71 & 1,89 & 2,03 & 2,02 & 2,00 & 4,01 & 0,09 \\
\hline
\end{tabular}

T1. $25 \mathrm{~g} / \mathrm{t}$ de pigmento industrial amarelo (carophyll yellow 10\%) + $20 \mathrm{~g} / \mathrm{t}$ de carophyll vermelho; T2. $300 \mathrm{~g} / \mathrm{t}$ de pigmento natural amarelo (Marigold 2\%) + $10 \mathrm{~g} / \mathrm{t}$ de vermelho industrial (Cantaxantina 10\%); T3. $300 \mathrm{~g} / \mathrm{t}$ de pigmento natural amarelo (Marigold 2\%) + $20 \mathrm{~g} / \mathrm{t}$ de vermelho industrial (Cantaxantina 10\%); T4. $300 \mathrm{~g} / \mathrm{t}$ de pigmento natural amarelo (Marigold $2 \%$ ) $+30 \mathrm{~g} / \mathrm{t}$ de vermelho industrial (Cantaxantina 10\%); T5. $300 \mathrm{~g} / \mathrm{t}$ de pigmento natural amarelo (Marigold $2 \%)+20 \mathrm{~g} / \mathrm{t}$ carophyll vermelho. CA - conversão alimentar.

Observa-se na Tabela 3 que não houve efeito $(p>0,05)$ dos tratamentos sobre as variáveis de qualidade interna e externa dos ovos, com exceção para a coloração da gema $(p<0,01)$ que foi maior para os tratamentos com inclusão de pigmentantes. 
Tabela 3. Qualidade interna e externa dos ovos de galinhas poedeiras comerciais recebendo dietas com diferentes níveis de suplementação de pigmentantes sintéticos e naturais em dietas a base de sorgo.

Table 3. Internal and external quality of eggs from commercial laying hens fed diets with different levels of supplementation of synthetic and natural pigments in sorghum-based diets.

\begin{tabular}{llllllll}
\hline \multirow{2}{*}{\multicolumn{1}{c}{ Variáveis }} & \multicolumn{7}{c}{ Tratamentos } \\
\cline { 2 - 7 } & T1 & T2 & T3 & T4 & T5 & CV & P-valor \\
\hline Peso Médio do Ovo (g) & 65,45 & 63,60 & 63,20 & 63,55 & 62,49 & 302 & 0,17 \\
Gravidade Específica $\left(\mathrm{g} / \mathrm{cm}^{3}\right)$ & 1,084 & 1,088 & 1,085 & 1,087 & 1,08 & 2,19 & 0,07 \\
Cor da Gema** & $10,61 \mathrm{e}$ & $11,00 \mathrm{~d}$ & $12,40 \mathrm{c}$ & $13,33 \mathrm{a}$ & $12,92 \mathrm{~b}$ & 2,14 & 0,02 \\
Gema (\%) & 25,23 & 25,18 & 25,01 & 24,99 & 24,95 & 3,26 & 0,06 \\
Casca (\%) & 9,36 & 9,84 & 9,64 & 9,57 & 9,78 & 2,87 & 0,08 \\
Albúmen (\%) & 65,39 & 64,96 & 65,33 & 65,42 & 65,25 & 6,53 & 0,14 \\
Espessura da Casca (mm) & 0,37 & 0,40 & 1,97 & 0,39 & 0,41 & 6,03 & 0,36 \\
Unidade Haugh & 76,85 & 77,23 & 76,91 & 77,10 & 77,51 & 1,24 & 0,45
\end{tabular}

T1: $25 \mathrm{~g} / \mathrm{t}$ de pigmento industrial amarelo (carophyll yellow 10\%) + $20 \mathrm{~g} / \mathrm{t}$ de carophyll vermelho; T2: $300 \mathrm{~g} / \mathrm{t}$ de pigmento natural amarelo (Marigold 2\%) + $10 \mathrm{~g} / \mathrm{t}$ de vermelho industrial (Cantaxantina 10\%); T3: $300 \mathrm{~g} / \mathrm{t}$ de pigmento natural amarelo (Marigold 2\%) $+20 \mathrm{~g} / \mathrm{t}$ de vermelho industrial (Cantaxantina 10\%); T4: $300 \mathrm{~g} / \mathrm{t}$ de pigmento natural amarelo (Marigold 2\%) + $30 \mathrm{~g} / \mathrm{t}$ de vermelho industrial (Cantaxantina 10\%); T5: $300 \mathrm{~g} / \mathrm{t}$ de pigmento natural amarelo (Marigold 2\%) +20 g/t carophyll vermelho. CV: Coeficiente de variação. ${ }^{* *}$ Média seguidas por letras diferentes na linha diferem estatísticamente pelo teste Tukey ao nível de $5 \%$ de probabilidade.

O nível de suplementação de $300 \mathrm{~g} / \mathrm{t}$ de pigmento natural amarelo + $30 \mathrm{~g} / \mathrm{t}$ de pigmento vermelho industrial em dietas para poedeiras proporcionou melhor índice de coloração da gema comparado aos demais tratamentos. O pior índice de coloração da gema foi obtido com a suplementação de $25 \mathrm{~g} / \mathrm{t}$ de pigmento industrial amarelo (carophyll yellow $10 \%$ ) $+20 \mathrm{~g} / \mathrm{t}$ de carophyll vermelho em dietas para poedeiras.

Conforme relatado por BITTENCOURT et al. (2019), a utilização de ingredientes alternativos ao milho nas rações, além de uma necessidade para reduzir custos na produção de ovos, é uma oportunidade para encontrar outras fontes energéticas que substituam o milho, sem ocorrer prejuízos no desempenho animal. Assim, o sorgo em dietas para poedeiras pode apresentar vantagens, pois é comercializado a um preço ao redor de $80 \%$ do preço do milho, apesar das diferenças nutricionais entre ambos (FASSANI et al. 2019).

Analisando os resultados, observa-se que o sorgo é considerado um alimento alternativo, porém seus grãos têm quantidade deficiente de caroteno e pigmentos como as xantofilas (MOURA et al. 2011), e por isso o seu fornecimento à ave induz à despigmentação da gema do ovo, o que pode ser resolvido com a adição de pigmentantes naturais ou sintéticos.

A utilização do pigmento natural não influenciou na conversão alimentar, produção de ovos e consumo de ração, o que corrobora com FASSANI et al. (2019) ao utilizar a cantaxantina na alimentação comercial em poedeiras de 55 semanas de idade. Do mesmo modo OLIVEIRA et al. (2021) não observaram efeito no desempenho de codornas japonesas alimentadas com dietas contendo extrato produzido a partir do resíduo da goiaba. NUNES JÚNIOR et al. (2020) também relatou que o uso de urucum e cenoura promove maior pigmentação das gemas de ovos de galinhas Hisex Brown e não prejudica o desempenho das aves. Os resultados deste ensaio estão de acordo com os resultados obtidos por MOURA et al. (2011) ao avaliar a utilização de pigmentantes naturais em rações baseadas em sorgo em codornas japonesas, não observando alterações no desempenho e qualidade dos ovos.

GARCIA et al. (2002) também encontraram resultados semelhantes ao analisar diferentes níveis do pigmento cantaxantina (0,12, 24, 48 e 60 ppm). Similarmente, VALENTIM et al. (2019) também não encontraram diferenças na utilização deste componente nesta variável, com exceção da coloração. De acordo com os autores, isto se deve ao fato deste pigmento apresentar capacidade química de pigmentação, mostrando-se um excelente corante para as gemas.

Para FASSANI et al. (2019), produtos à base de cantaxantina, carophyll vermelho são essenciais quando o objetivo é alteração da pigmentação da gema dos ovos. De maneira semelhante, MOURA et al. (2016) também observaram maiores escores de coloração com a utilização do pigmentante. 
BITTENCOURT et al. (2019) relataram em seu estudo com adição de grãos secos de destilaria na alimentação de codornas japonesas que o nível de 20 \% de DDGS apresentou uma coloração mais intensa da gema do ovo, podendo se considerar que, com a utilização do milho DDGS na ração das codornas, esta ficará mais amarela, melhorando a aceitação do consumidor.

A coloração da gema é muito utilizada pelos consumidores como uma ferramenta de análise de qualidade e a cor desejada varia entre os mercados. Além disso, trata-se de uma das características para avaliar a qualidade dos ovos, apresentando uma função importante no critério para escolha dos ovos pelo consumidor, sendo esta uma característica sensorial mais exigida (RADDATZ-MOTA et al. 2017).

Mas a coloração não condiz com o valor nutricional do ovo, como afirmam HERNANDEZ et al. (2000). Outro ponto relevante é que, apesar de alguns carotenóides serem precursores da vitamina $A$, de acordo com PÉREZ-VENDRELL et al. (2001), isso não refletiu em melhora significativa no desempenho das aves.

VALENTIM et al. (2020) trabalhando com pigmentantes naturais à base de extratos de páprica e calêndula na dieta de codornas mostraram que a adição de diferentes pigmentos na ração de codornas não influenciou no desempenho e na qualidade dos ovos, mas pode ser uma alternativa para evitar o uso de pigmentos sintéticos por apresentar uma gema colorida, valiosa para o consumidor. Outro trabalho relata a ação de fontes vegetais como agente pigmentante, como demonstrado por SILVA et al. (2019) que concluíram que a suplementação alimentar com folha de bananeira na dieta de poedeiras Carijó do sistema Cage-free aumenta a pigmentação da gema do ovo.

Devido às exigências de mercado, os pigmentantes naturais têm sido cada vez mais utilizados para potencializar a cor das gemas dos ovos, gerando mais opções de aditivos e de insumos como o sorgo visando diminuição dos custos de produção e garantindo a produtividade animal.

\section{CONCLUSÃO}

Pigmentos naturais associados ou não a pigmentos sintéticos não interferem no desempenho e na qualidade interna e externa dos ovos, com exceção da coloração da gema, onde o nível de suplementação de 300 g.t $\mathrm{t}^{-1}$ de pigmento natural amarelo $+30 \mathrm{~g} \cdot \mathrm{t}^{-1}$ de pigmento vermelho industrial em dietas para poedeiras proporcionou melhor índice de coloração da gema.

\section{REFERÊNCIAS}

ABPA. 2020. Associação Brasileira de Proteína Animal. Relatório Anual 2020. Ovos. São Paulo. 154.

ALLEONI ACC \& ANTUNES AJ. 2001. Unidade Haugh como medida da qualidade de ovos de galinha armazenados sob refrigeração. Scientia Agricola 58: 681-685.

BITTENCOURT TM et al. 2019. Distillers dried grains with solubles from corn in diet of japanese quails. Acta Scientiarum. Animal Sciences 41: 1-7.

FASSANI EJ et al. 2019. Coloração de gema de ovo de poedeiras comerciais recebendo pigmentante comercial na ração. Ciência Animal Brasileira 20: 1-10.

FREITAS ER et al. 2004. Comparação de métodos de determinação da gravidade específica de ovos de poedeiras comerciais. Pesquisa Agropecuária Brasileira 39: 509-512.

GALOBART J et al. 2004. Egg yolk color as affected by saponified oleoresin of red pepper (Capsicum annuum) fed to laying hens. Journal of Applied Poultry Research 13: 328-334

GARCIA EA et al. 2002. Efeito dos níveis de cantaxantina na dieta sobre o desempenho e qualidade dos ovos de poedeiras comerciais. Revista Brasileira de Ciência Avícola 4: 1-7.

HERNANDEZ J et al. 2000. Perceptions of egg quality in Europe. International Poultry Production 8: 1-11.

LANA SRV et al. 2017. Qualidade de ovos de poedeiras comerciais armazenados em diferentes temperaturas e períodos de estocagem. Revista Brasileira de Saúde e Produção Animal 18: 140-151.

LOPES IRV et al. 2011. Desempenho e qualidade dos ovos de poedeiras comerciais alimentadas com rações contendo farelo de coco tratado ou não com antioxidante. Revista Brasileira de Zootecnia 40: 2431-2438.

MOURA AMAM et al. 2011. Pigmentantes naturais em rações à base de sorgo para codornas japonesas em postura. Revista Brasileira de Zootecnia 40: 2443-2449.

MOURA AMA et al. 2016. Synthetic pigments for Japanese quail fed diets with sorghum. Arquivo Brasileiro de Medicina Veterinária e Zootecnia 68: 1007-1014.

NUNES JUNIOR DA et al. 2020. Vegetable pigments in sorghum-based diets for laying hens. Caderno de Ciências Agrárias 12: 1-6.

OLIVEIRA HF et al. 2021. Egg characteristics of Japanese quail fed diets containing guava extract (Psidium guajava L.). Acta Scientiarum: Animal Sciences 43: 1-7.

OLIVEIRA HF et al. 2021. Lipid stability of egg and laying performance of Japanese quail feed with extract of guava (Psidium guajava L.). Anais da Academia Brasileira de Ciências 93: 1-12.

PÉREZ-VENDRELL AM et al. 2001. Influence of source and ratio of xanthophyll pigments on broiler chicken pigmentation and performance. Poultry Science 80: 320-326. 
RADDATZ-MOTA D et al. 2017. Achiote (Bixa orellana L.): a natural source of pigment and vitamin E. Journal of Food Science and Technology 54: 1729-1741.

SEIBEL NF et al. 2010. Caracterização sensorial de ovos de codornas alimentadas com dietas modificadas. Food Science and Technology 30: 884-889.

VALENTIM JK et al. 2020. Natural and synthetic pigments in diet of Japanese quails. Acta Scientiarum. Animal Sciences 42: 1-6.

VALENTIM JK et al. 2019. Pigmentantes vegetais e sintéticos em dietas de galinhas poedeiras Negras. Boletim de Indústria Animal 76: 1-9. 